\title{
Prevalência e fatores de risco para a leptospirose em bovinos de Mato Grosso do Sul ${ }^{1}$
}

\author{
Aline de O. Figueiredo ${ }^{2 *}$, Aiesca O. Pellegrin ${ }^{3}$, Vitor S.P. Gonçalves ${ }^{4}$, Emanuel \\ B. Freitas ${ }^{5}$, Letícia Almeida R.C. Monteiro ${ }^{5}$, Jacqueline M. de Oliveira ${ }^{5}$ e Ana \\ Luiza A.R. Osório6
}

\begin{abstract}
Figueiredo A.O., Pellegrin A.O., Gonçalves V.S.P., Freitas E.B., Monteiro L.A.R.C., Oliveira J.M. \& Osório A.L.A.R. 2009. [Prevalence and risk factors for bovine leptospirosis in Mato Grosso do Sul, Brazil.] Prevalência e fatores de risco para a leptospirose em bovinos do Mato Grosso do Sul. Pesquisa Veterinária Brasileira 29(5):375381. Programa Mestrado em Ciência Animal, Universidade Federal de Mato Grosso do Sul, Campo Grande, MS 79070-900, Brazil. E-mail: line_figueiredo@yahoo.com.br

The prevalence of anti-Leptospira spp. antibodies was estimated for female cattle aged 24 months or older. The sample comprised 178 herds from 22 counties in the state of Mato Grosso do Sul, Brazil. The risk factors associated with the presence of infeccion were investigated. A total of 2,573 blood serum samples were tested against 10 leptospira serovars using the microagglutination test (MAT). Titers of 100 or higher for one or more serovars were detected in 1,801 females (98.8\%) from 161 herds (96.5\%). Serovar Hardjo $(65.6 \%)$ was the most frequent, followed by serovar Wolffi $(12.3 \%)$. These results suggest that bovine leptospirosis is widespread in all the counties under study, with a high prevalence both at the animal and the herd level. Beef farms and the Zebu breed were associated to the higher risk of herd infection by leptospiras.
\end{abstract}

INDEX TERMS: Leptospirosis, cattle, epidemiology, herd prevalence.

RESUMO.- Foi investigada a prevalência de anticorpos antileptospira em fêmeas bovinas com idade igual ou superior a 24 meses, provenientes de 178 rebanhos de 22 municípios do estado de Mato Grosso do Sul, bem como identificados fatores de risco associados à infecção. Foram analisadas 2.573 amostras de soro sangüíneo por

\footnotetext{
${ }^{1}$ Recebido em 18 de julho de 2008.

Aceito para publicação em 9 de dezembro de 2008.

2 Mestranda do Programa de Pós-Graduação em Ciência Animal, Universidade Federal de Mato Grosso do Sul (UFMS), Cidade Universitária, Campo Grande, MS 79070-900, Brasil. *Autor para correspondência: line_figueiredo@yahoo.com.br

${ }^{3}$ Embrapa Pantanal, Rua 21 de setembro 1880, Corumbá, MS 79320900, Brasil.

${ }^{4}$ Curso de Medicina Veterinária, Faculdade de Agronomia e Medicina Veterinária, Universidade de Brasília, Campus Darcy Ribeiro, Asa Norte, Brasília, DF 70910-900, Brasil.

${ }^{5}$ Agência Estadual de Defesa Sanitária Animal e Vegetal (lagro/MS), Av. Senador Filinto Muller 1146, Bairro Universitário, Campo Grande, MS 79074-902.

${ }^{6}$ Docente do Programa de Pós-Graduação em Ciência Animal, UFMS, Campo Grande, MS.
}

meio do teste de soroaglutinação microscópica perante 10 sorovares de leptospira. Títulos iguais ou superiores a 100 para um ou mais sorovares foram detectados em 1.801 fêmeas $(98,8 \%)$ de 161 (96,5\%) rebanhos. O sorovar Hardjo $(65,6 \%)$ foi apontado como o mais provável, seguido do sorovar Wolffi (12,3\%). Os resultados demonstram que a leptospirose bovina se encontra presente em todos os municípios estudados, com alta prevalência, tanto em animais como em rebanhos. Os fatores de risco identificados neste estudo e associados à infecção por bactérias do gênero lepstopira foram o tipo de exploração pecuária de corte e a raça Zebu.

TERMOS DE INDEXAÇÃO: Leptospirose, bovinos, epidemiologia, prevalência de rebanhos.

\section{INTRODUÇÃO}

A leptospirose é uma doença infecto-contagiosa que acomete animais e humanos, causada por qualquer espécie patogênica de bactérias do gênero Leptospira spp. (OIE 2006). Já foi diagnosticada em todos os continentes, no entanto, na América Latina, África e Ásia, os níveis de 
ocorrência são elevados, pois as condições ambientais favorecem a persistência e a disseminação do agente (Blenden 1976).

Na saúde animal, as conseqüências desta infecção são de esfera econômica, tendo em vista o envolvimento de animais de produção como bovinos, ovinos, caprinos, suínos e eqüinos (Brasil 1995). Nos bovinos, as perdas econômicas decorrem de transtornos reprodutivos como infertilidade, aborto, nascimento de bezerros fracos e diminuição temporária da produção leiteira (Cervantes et al. 2002).

No Brasil, a soroprevalência da leptospirose em rebanhos varia de $74 \%$ a $100 \%$ (Homem et al. 2001, Favero et al. 2001, Thompson et al. 2006, Lage et al. 2007) e em animais de $45,56 \%$ a $62,3 \%$ (Langoni et al. 2000, Favero et al. 2001). Os inquéritos sorológicos realizados em populações bovinas, no território brasileiro, evidenciam como importantes os sorovares Hardjo, Wolffi, Pomona, Grippotyphosa, Icterohaemorrhagiae e Canicola, sendo mais prevalente o sorovar Hardjo (Favero et al. 2001, Lilenbaum \& Souza 2003, Araújo et al. 2005, Thompson et al. 2006, Lage et al. 2007). No Mato Grosso do Sul, o sorovar Hardjo também é o mais freqüente (Madruga et al. 1980, Pellegrin et al. 1999, Favero et al. 2001).

O objetivo deste estudo foi determinar a prevalência de anticorpos anti-Leptospira spp. em fêmeas bovinas em idade reprodutiva no Estado do Mato Grosso do Sul, bem como identificar os fatores de risco associados à infecção.

\section{MATERIAL E MÉTODOS}

\section{Área de estudo}

A investigação foi realizada numa região do estado de Mato Grosso do Sul (MS) composta por 22 municípios, que constitui uma área de $70.215 \mathrm{~km}^{2}$, o que representa 19,7\% do MS (Brasil 2002). Esses municípios fazem parte de três das quatro mesorregiões do estado (Mesorregião do Centro Norte de MS; Mesorregião do Leste de MS; e Mesorregião do Sudoeste de MS) (Fig.1). O rebanho bovino da região estudada é de aproximadamente 5,7 milhões de cabeças, com média de 321 cabeças por rebanho, sendo a maioria com aptidão produtiva de corte, correspondentes a $23,2 \%$ do rebanho total do estado (Brasil 2003), que em 2004 era de 24,715 milhões de bovinos (Brasil 2004).

\section{Amostragem}

Para calcular o tamanho da amostra destinada a estimar prevalência de rebanhos considerou-se o grau de confiança de $95 \%$ e o nível de precisão absoluta dos resultados de $\pm 5 \%$ (Noordhuizen et al.1997). A prevalência esperada de rebanhos, utilizada no cálculo foi de $87 \%$, com base nos achados sorológicos de Favero et al. (2001), em rebanhos de 21 estados do Brasil. Assim, seria necessário amostrar 174 rebanhos de acordo com a fórmula padrão para amostragem aleatória simples (Thrusfield 1995, Noordhuizen et al. 1997), porém foi possível amostrar 178 rebanhos.

Para o sorteio das propriedades foi utilizada a listagem das fichas sanitárias fornecidas pela Agência Estadual de Defesa Sanitária Animal e Vegetal (lagro/MS) e para o cálculo do intervalo amostral dividiu-se o número total de propriedades pelo número de propriedades a serem amostradas. Nas propriedades rurais, nas quais claramente foram identificados mais de um rebanho, escolheu-se como alvo do estudo o rebanho bovino de maior importância econômica, no qual os animais estavam submetidos ao mesmo manejo. Se uma propriedade sorteada não pôde, por quaisquer motivos, ser visitada, foi substituída por outra, nas proximidades e com as mesmas características de produção.

Foram simulados diversos cenários, com auxilio do software Herdacc $^{\circledR}$, com o objetivo de determinar o número de fêmeas adultas a serem amostradas por rebanho. Para tanto, considerou-se que a especificidade e sensibilidade agregadas, ou em nível de rebanho, deveriam ser iguais ou superiores à sensibilidade e especificidade do teste diagnóstico, que neste caso

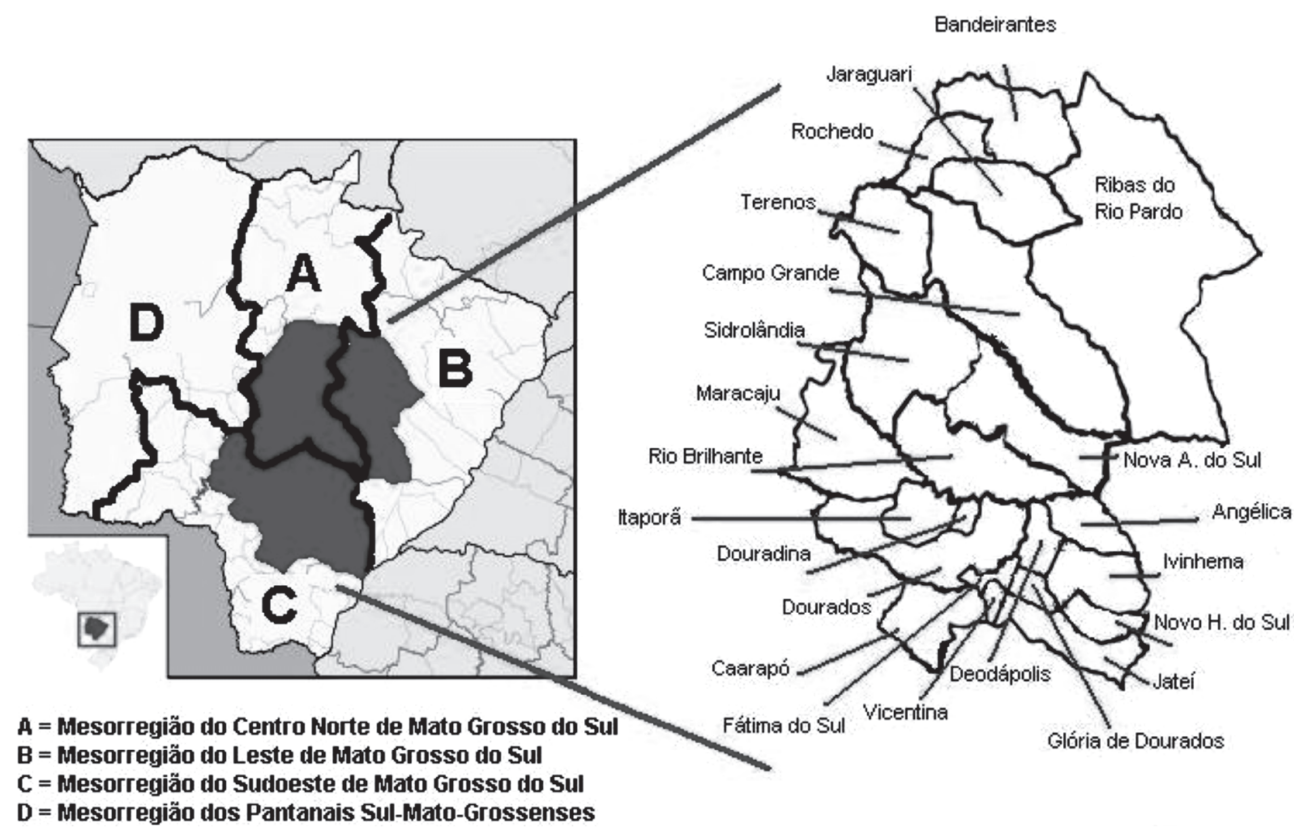

Fig.1. Municípios que compõem a região do estudo. 
eram respectivamente de 0,82 e 0,97 . Simularam-se cinco cenários de prevalência intra-rebanho $(50 \%, 70 \%, 80 \%, 85 \%$ e $90 \%$ ), além do cenário base em que o rebanho estaria livre de infecção. Foram simulados vários pontos de corte, ou número de animais positivos, para classificar o rebanho como infectado. O número de animais no rebanho foi de 300 . Os resultados das simulações permitiram estabelecer uma amostra de 14 fêmeas adultas e fixar o ponto de corte em quatro animais por rebanho, ou seja, rebanhos com quatro ou mais animais positivos seriam considerados infectados. Os rebanhos com um, dois ou três animais reagentes seriam inconclusivos, enquanto os rebanhos sem reações positivas seriam negativos. Este método visa garantir que a especificidade agregada seja boa, evitando assim a sobreestimativa de prevalência de rebanho devido à classificação de muitos rebanhos falso-positivos. Por outro lado, a sensibilidade agregada foi mantida em valores aceitáveis apesar do ponto de corte utilizado em razão da alta prevalência intra-rebanho encontrada na literatura.

A seleção das fêmeas foi feita de forma aleatória, empregando-se o método de amostragem aleatória simples ou sistemática. A escolha por um dos métodos foi definida dividindo o total de fêmeas com idade igual ou superior a dois anos existentes na propriedade pelo total de fêmeas a serem amostradas. Para resultados inferiores a dois, foi empregado o método de amostragem aleatória simples, consultando uma tabela de números aleatórios; nos casos em que o resultado era superior a dois, foi empregado o método de amostragem aleatória sistemática. No total, foram amostradas 2.573 fêmeas.

\section{Obtenção das amostras}

As amostras foram colhidas no período de dezembro de 2003 a março de 2004 e transportadas para o Laboratório de Diagnóstico de Doenças Animais (Laddan) da lagro. Os soros obtidos foram estocados a $-20^{\circ} \mathrm{C}$ e, depois, submetidos ao teste de diagnóstico de leptospirose.

\section{Teste diagnóstico}

Para o diagnóstico foi empregado o teste de soroaglutinação microscópica (SAM), utilizando uma coleção de antígenos vivos composta de 10 sorovares: Icterohaemorrhagiae, Copenhageni, Canicola, Autumnalis, Pomona, Grippotyphosa, Hebdomadis, Wolffi, Bratislava e Hardjo. Foram consideradas reagentes as amostras com título igual ou superior a 100 , com $50 \%$ de aglutinação ou desaparecimento das células do campo, em microscopia de campo escuro (Brasil 1995). Os soros reagentes foram titulados em séries geométricas de quatro diluições de razão dois, sendo o título dado como a recíproca da maior diluição em que houve aglutinação.

Para efeito do cálculo de prevalência considerou-se qualquer animal reagente para um ou mais sorovares. Para a determinação dos sorovares predominantes foram considerados aqueles de maior título e os animais que apresentaram dois ou mais sorovares com títulos idênticos foram excluídos dessa análise. No entanto, esses animais foram computados como reatores para pelo menos um sorovar no cálculo de prevalência.

\section{Análise estatística}

Prevalência de animais infectados. As prevalências aparente $(\mathrm{Pa})$ e real $(\mathrm{Pr})$ para animais foram calculadas conforme Noordhuizen et al. (1997) e Bennett et al. (1991). A $\mathrm{Pa}$ foi obtida ponderando-se a frequência com o peso que cada propriedade tem em número de animais em relação ao total de propriedades (fator de ponderação). Os valores de $\mathrm{Pr}$ foram estimados ajustando-se o valor da Pa para a especificidade $(0,97)$ e sensibilidade $(0,82)$ do teste de SAM (Cumberland et al. 1999).

$P a=\Sigma w_{i} y_{i} / \Sigma w_{i} x_{i}$, onde:

$w$ = fator de ponderação;

$\mathrm{x}=$ número de animais testados por rebanho;

$\mathrm{y}=$ número de animais positivos;

$\mathrm{i}$ = número de cada rebanho.

$\operatorname{Pr}=(\mathbf{P a}+$ Esp-1) $/($ Sen + Esp-1), onde:

Sen $=$ sensibilidade do teste;

$\mathrm{Pa}=$ prevalência aparente;

$\mathrm{Esp}=$ especificidade do teste.

Prevalência de rebanhos infectados. A prevalência aparente de rebanho (PaR) (Martin et al. 1987) foi estimada por meio da seguinte fórmula:

$\mathrm{PaR}=\mathrm{n} / \mathrm{N}$, onde:

$\mathrm{n}=$ número de rebanhos classificados como positivos;

$\mathrm{N}=$ número total de rebanhos testados.

Prevalência real de rebanho (PrR). Foi calculada de acordo com Martin et al. (1992), utilizando a seguinte fórmula:

PrR = (PaR + EspR-1) / (SenR + EspR-1), onde:

$\operatorname{PrR}=$ prevalência real de rebanho;

$\mathrm{PaR}=$ prevalência aparente de rebanho;

EspR = especificidade de rebanho;

SenR = sensibilidade de rebanho.

Os intervalos de confiança (IC) das prevalências aparente e real de animais e de rebanhos foram calculados para uma confiança de 95\%, conforme Martin et al. (1987), por meio da seguinte fórmula:

IC = valor estatístico $\pm(z$ * EP $)$, onde:

$z=1,96$ (Sampaio 2007);

$\mathrm{EP}=$ erro-padrão.

Para o cálculo do erro padrão da prevalência de animais foi utilizada a fórmula abaixo, assumindo-se que a amostragem de animais foi do tipo conglomerado (Bennett et al. 1991):

$$
\begin{aligned}
& \text { EPconglomerado }=\left(C / \Sigma w_{i} x_{i}\right)^{*} \sqrt{ }\left\{\left[\left(\Sigma w_{i}^{2} y_{i}^{2}\right)-\left(2 P a \Sigma w_{i}^{2} x_{i} y_{i}\right)+\right.\right. \\
& \left.\left.\left(\mathrm{Pa}^{2} \boldsymbol{\Sigma} \mathrm{w}_{\mathrm{i}}^{2} \mathbf{x}_{\mathrm{i}}^{2}\right)\right] /\left[\mathrm{C}^{*}(\mathbf{C}-1)\right]\right\} \text { onde: } \\
& \mathrm{C}=\text { número do conglomerado, ou seja, o número de }
\end{aligned}
$$

O erro padrão da prevalência de rebanhos é calculado utilizando-se a seguinte fórmula:

EP $\mathrm{Pa}$ rebanho= $\sqrt{ } \mathrm{Pa}$ rebanho*(1-Pa rebanho)/total de propriedades examinadas. 
Estimativa da sensibilidade e especificidade de rebanho. A SenR e EspR foram estimadas de acordo com Dohoo et al. (2003). Considerou-se o ponto de corte igual a quatro, significando que o achado de pelo menos quatro animais reagentes no rebanho o classificava como rebanho positivo, de um a três animais como rebanho inconclusivo e nenhum animal regente (zero) como negativo. Como a fração de animais amostrados no rebanho foi em geral inferior a $10 \%$, utilizou-se a distribuição binomial com auxilio do software Herdaac®, especifico para esse tipo de cálculo amostral.

Valores preditivos. $O$ valor preditivo positivo (VPP) e o valor preditivo negativo (VPN) do diagnóstico do rebanho foram estimados de acordo com Noordhuizen et al. (1997) e Dohoo et al. (2003), por meio das seguintes fórmulas:

VPP $=[P r R *$ SenR $] /[[(\operatorname{PrR}$ * SenR $)+(1-\operatorname{PrR})] *(1-$ EspR $)]$ VPN $=[(1-\operatorname{PrR})$ * EspR $] /\left[\left[\operatorname{PrR}{ }^{*}(1-\operatorname{SenR})\right]+[(1-\mathrm{PrR})\right.$ * EspR]], onde:

$\operatorname{PrR}=$ prevalência real de rebanho;

SenR = sensibilidade de rebanho;

$\mathrm{EspR}=$ especificidade de rebanho.

\section{Questionário e análise dos fatores de risco}

Foi aplicado um questionário aos proprietários ou responsáveis pelos rebanhos, no qual foram incluídas informações do tipo de exploração (corte, leite e misto), uso de inseminação artificial, raças predominantes, presença de suínos, compra e venda de animais e áreas alagadiças. Foi realizado estudo de fatores de risco do tipo transversal. Para tanto foram formados dois grupos de propriedades (positivas e negativas) que, quando comparados entre si quanto às variáveis pesquisadas no questionário epidemiológico, permitiu medir a força da associação dessas variáveis com a presença da leptospirose.

As variáveis de risco associadas à soropositividade do rebanho para pelo menos um sorovar foram analisadas por meio da estimativa pontual e intervalar da odds ratio (OR), com intervalo de confiança de $95 \%$. O teste de hipóteses foi realizado com o teste de qui-quadrado ou teste exato de Fisher, quando indicado (Sampaio 2007).

O resultado do teste de diagnóstico e as informações do questionário aplicado em cada propriedade foram armazenados em um banco de dados, utilizando o programa Microsoft Access ${ }^{\circledR}$.

\section{RESULTADOS}

De acordo com o questionário, observou-se que das 178 propriedades, $71(39,9 \%)$ eram destinadas à exploração de corte, $81(45,5 \%)$ leiteira e $26(14,6 \%)$ de criação mista. Das propriedades amostradas, constatou-se que em nenhuma delas havia orientação para vacinação antileptospirose.

O Quadro 1 apresenta os resultados do teste de SAM em rebanhos e animais amostrados nos diferentes municípios da área de estudo.

A Figura 2 ilustra o percentual de reações por sorovar na área estudada, considerando apenas os reagentes mais prováveis, analisados por meio do teste SAM, com ponto de corte igual ao título de 100.

O Quadro 2 registra os resultados de prevalência aparente, sensibilidade e especifidade de rebanho, prevalên-
Quadro 1. Frequência de anticorpos anti-Leptospira spp., determinados pelo teste de SAM, em animais e rebanhos de Mato Grosso do Sul

\begin{tabular}{|c|c|c|c|c|c|c|}
\hline \multirow[t]{2}{*}{ Municípios } & \multicolumn{3}{|c|}{ Rebanhos } & \multicolumn{3}{|c|}{ Bovinos } \\
\hline & $\begin{array}{l}\text { Exami- } \\
\text { nados }\end{array}$ & $\begin{array}{l}\text { Posi- } \\
\text { tivos }\end{array}$ & $\begin{array}{c}\text { Freq. } \\
(\%)\end{array}$ & $\begin{array}{l}\text { Exami- } \\
\text { nados }\end{array}$ & $\begin{array}{l}\text { Posi- } \\
\text { tivos }\end{array}$ & $\begin{array}{l}\text { Freq. } \\
(\%)\end{array}$ \\
\hline Angélica & 4 & 3 & $75,0 \%$ & 49 & 20 & $40,8 \%$ \\
\hline Bandeirantes & 7 & 7 & $100,0 \%$ & 121 & 97 & $80,2 \%$ \\
\hline Caarapó & 7 & 7 & $100,0 \%$ & 113 & 70 & $61,9 \%$ \\
\hline Campo Grande & 15 & 15 & $100,0 \%$ & 208 & 162 & $77,9 \%$ \\
\hline Deodápolis & 8 & 7 & $87,5 \%$ & 97 & 68 & $70,1 \%$ \\
\hline Douradina & 2 & 1 & $50,0 \%$ & 22 & 9 & $40,9 \%$ \\
\hline Dourados & 16 & 13 & $81,3 \%$ & 201 & 145 & $72,1 \%$ \\
\hline Fátima & 4 & 3 & $75,0 \%$ & 43 & 23 & $53,5 \%$ \\
\hline Glória de Dourados & 9 & 9 & $100,0 \%$ & 120 & 82 & $68,3 \%$ \\
\hline Itaporã & 6 & 6 & $100,0 \%$ & 74 & 49 & $66,2 \%$ \\
\hline Ivinhen & 13 & 11 & $84,6 \%$ & 166 & 83 & $50,0 \%$ \\
\hline Jaraguari & 7 & 7 & $100,0 \%$ & 91 & 76 & $83,5 \%$ \\
\hline Jateí & 7 & 6 & $85,7 \%$ & 102 & 68 & $66,7 \%$ \\
\hline Maracaju & 5 & 5 & $100,0 \%$ & 83 & 78 & $94,0 \%$ \\
\hline Nova Alvorada do Sul & 8 & 8 & $100,0 \%$ & 160 & 129 & $80,6 \%$ \\
\hline Novo Horizonte do Sul & 10 & 9 & $90,0 \%$ & 158 & 96 & $60,8 \%$ \\
\hline Ribas do Rio Pardo & 17 & 13 & $76,5 \%$ & 266 & 141 & $53,0 \%$ \\
\hline Rio Brilhante & 6 & 6 & $100,0 \%$ & 88 & 67 & $76,1 \%$ \\
\hline Rochedo & 5 & 5 & $100,0 \%$ & 90 & 83 & $92,2 \%$ \\
\hline Sidrolândia & 8 & 7 & $87,5 \%$ & 88 & 64 & $72,7 \%$ \\
\hline Terenos & 10 & 10 & $100,0 \%$ & 172 & 158 & $91,9 \%$ \\
\hline Vicentina & 4 & 3 & $75,0 \%$ & 61 & 33 & $54,1 \%$ \\
\hline Total & 178 & 161 & $90,4 \%$ & 2573 & 1801 & $70,0 \%$ \\
\hline
\end{tabular}

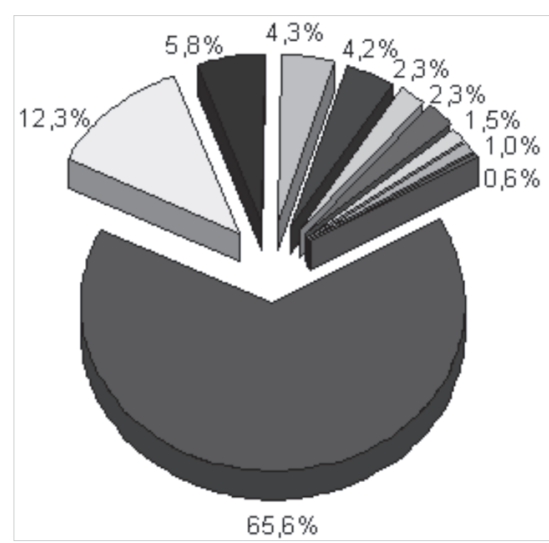

- HARDJO

口WOLFI

- ICTERoHaEM ORRHAGIAE 口GRIPPOTYPHOSA

- BRATISLAVA

口HEBDOMADIS

- CANICOLA

$\square$ COPENHAGENN

$\triangle P O M O N A$

- AUTUMNALIS

Fig.2. Percentual de amostras reagentes, segundo os sorovares mais prováveis, analisadas por meio do teste de soroaglutinação microscópica em 1.380 amostras.

Quadro 2. Prevalência aparente, intervalo de confiança, sensibilidade e especifidade de rebanho, prevalência real e valores preditivos de leptospirose bovina em rebanhos

\begin{tabular}{|c|c|c|c|c|c|c|}
\hline $\begin{array}{c}\text { PaR IC inferior } \\
\mathrm{PaR}\end{array}$ & $\begin{array}{l}\text { IC superior } \\
\text { PaR }\end{array}$ & SenR & EspR & PrR & VPP & VPN \\
\hline $85,8 \%$ & $95,1 \%$ & $93,7 \%$ & $99,9 \%$ & $96,5 \%$ & $99,99 \%$ & $62,74 \%$ \\
\hline
\end{tabular}

$\mathrm{PaR}=$ prevalência aparente de rebanhos; IC = intervalo de confiança; SenR = sensibilidade de rebanho; EspR = especificidade de rebanho; $\operatorname{PrR}=$ prevalência real de rebanho; VPP = valor preditivo positivo; VPN $=$ valor preditivo negativo.

cia real e valores preditivos de leptospirose bovina em rebanhos. No Quadro 3 observa-se os valores de prevalência aparente e prevalência real de leptospirose bovina 
Quadro 3. Prevalência aparente, intervalo de confiança e prevalência real de leptospirose bovina em animais

\begin{tabular}{cccc}
\hline $\mathrm{Pa}$ & $\mathrm{IC}$ inferior $\mathrm{Pa}$ & IC superior $\mathrm{Pa}$ & $\mathrm{Pr}$ \\
\hline $81,1 \%$ & $76,3 \%$ & $85,9 \%$ & $98,8 \%$ \\
\hline
\end{tabular}

$\mathrm{Pa}=$ prevalência aparente; $\mathrm{IC}=$ intervalo de confiança; $\mathrm{Pr}=$ obtida com base na correção da Pa para sensibilidade e especificidade do teste.

Quadro 4. Variáveis de risco para leptospirose nos rebanhos considerando as reações para pelo menos um sorovar, com os correspondentes valores de odds ratio (OR)

\begin{tabular}{|c|c|c|c|c|c|c|}
\hline Variáveis & Descrição & $\begin{array}{l}\text { Posi- } \\
\text { tivos }\end{array}$ & $\begin{array}{l}\text { Nega- } \\
\text { tivos }\end{array}$ & OR & $\begin{array}{c}\mathrm{IC}^{\mathrm{a}} \\
\text { superior }\end{array}$ & $\begin{array}{c}\text { IC } \\
\text { inferior }\end{array}$ \\
\hline \multirow[t]{6}{*}{ Exploração } & Leite $(85,2 \%)^{b}$ & 69 & 12 & & & \\
\hline & Mista $(84,6 \%)$ & 22 & 4 & 1,05 & 0,30 & 3,59 \\
\hline & Corte (98,6\%) & 70 & 1 & & & \\
\hline & Mista (84,6\%) & 22 & 4 & $12,73^{*}$ & 2,07 & 78,35 \\
\hline & Corte $(98,6 \%)$ & 70 & 1 & & & \\
\hline & Leite $(85,2 \%)$ & 69 & 12 & $12,17^{\star *}$ & 2,23 & 64,46 \\
\hline \multirow[t]{2}{*}{$\begin{array}{l}\text { Ingresso } \\
\text { de animais }\end{array}$} & $\begin{array}{l}\text { Compra animais } \\
(92,3 \%)\end{array}$ & 72 & 6 & & & \\
\hline & $\begin{array}{l}\text { Não compra ani- } \\
\text { mais }(89,0 \%)\end{array}$ & 89 & 11 & 1,48 & 0,52 & 4,20 \\
\hline \multirow[t]{2}{*}{$\begin{array}{c}\text { Fonte de } \\
\text { contaminação }\end{array}$} & $\begin{array}{l}\text { Existe área ala- } \\
\text { gadiça }(90,2 \%)\end{array}$ & 119 & 13 & & & \\
\hline & $\begin{array}{l}\text { Não existe } \\
(91,3 \%)\end{array}$ & 42 & 4 & 0,87 & 0,27 & 2,83 \\
\hline \multirow[t]{2}{*}{ Reprodução } & Touro (89,9\%) & 142 & 16 & & & \\
\hline & $\begin{array}{l}\text { Inseminação ar- } \\
\text { tificial e touro } \\
(93,8 \%)\end{array}$ & 15 & 1 & 0,59 & 0,07 & 4,70 \\
\hline \multirow[t]{2}{*}{ Suínos } & $\operatorname{Sim}(88,3 \%)$ & 91 & 12 & & & \\
\hline & Não $(93,3 \%)$ & 70 & 5 & 0,54 & 0,18 & 1,59 \\
\hline \multirow[t]{6}{*}{ Raça } & Mestiço $(85,0 \%)$ & 68 & 12 & & & \\
\hline & $\begin{array}{l}\text { Europeu de leite } \\
(82,6 \%)\end{array}$ & 19 & 4 & 1,19 & 0,34 & 4,15 \\
\hline & Zebu $(98,5 \%)$ & 67 & 1 & & & \\
\hline & $\begin{array}{l}\text { Europeu de leite } \\
(82,6 \%)\end{array}$ & 19 & 4 & $14,11^{*}$ & 2,33 & 85,40 \\
\hline & Zebu $(98,5 \%)$ & 67 & 1 & & & \\
\hline & Mestiço $(85,0 \%)$ & 68 & 12 & $11,82^{\star *}$ & 2,21 & 63,21 \\
\hline
\end{tabular}

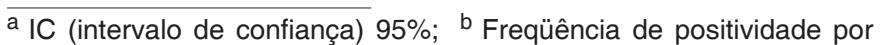
categoria; * Teste exato de Fisher $p<0,05$; ${ }^{* *}$ Teste de $\chi 2 ; p<0,05$.

em animais, na área estudada no estado do Mato Grosso do Sul.

As análises das variáveis de risco associadas à soropositividade dos rebanhos para pelo menos um sorovar, por meio da análise pontual e intervalar da odds ratio, identificaram como fatores de risco as variáveis raça (zebu) e tipo de exploração (corte) (Quadro 4).

\section{DISCUSSÃO}

Rebanhos e animais sororreagentes para Leptospira spp. foram detectados nos 22 municípios estudados (Quadro 1). Resultado semelhante foi observado por Favero et al. (2001) no estado do Mato Grosso do Sul, quando 100\% dos municípios apresentaram pelo menos um animal sororreagente em todos os rebanhos amostrados, embora em municípios diferentes dos deste estudo.
A prevalência aparente de $90,4 \%(85,8 \%-95,1 \%)$ de sororreação para Leptospira spp. dos rebanhos estudados (Quadro 2), assemelha-se às encontradas por Homem et al. (2001) no município de Uruará, Pará, de 97\% (90,9\%-99,5\%) e por Aguiar et al. (2006), no município de Monte Negro, Rondônia, de 95,3\% (88,5\%-98,7\%). No município de Garanhuns, estado do Pernambuco, Oliveira et al. (2001) testaram rebanhos leiteiros e encontraram pelo menos um animal sororreagente em todas as propriedades analisadas. Também em rebanhos leiteiros, no estado do Rio de Janeiro, Lilenbaum \& Santos (1995) constataram prevalência de $80,95 \%$. Em estudo realizado por Favero et al. (2001) em 21 estados brasileiros, a prevalência de propriedades com pelo menos um animal positivo foi de $84,1 \%$, com valores variando entre $74 \%$ e $100 \%$, incluindo rebanhos positivos em todos os estados analisados. Cabe lembrar que apesar de neste estudo ter sido aplicado critério de maior especificidade para classificar a propriedade como positiva, ao definir o ponto de corte igual a quatro, a prevalência de rebanho foi muito alta, em virtude da prevalência intra-rebanho também ser muito alta. Este dado demonstra que a leptospirose se dissemina muito rapidamente após ser introduzida em plantéis bovinos, como já havia sido sugerido no modelo analítico desenvolvido por Thompson et al. (2006).

Em animais, a prevalência aparente obtida de $81,1 \%$ (Quadro 3) foi superior a encontrada no estudo de Favero et al. (2001) nos 21 estados brasileiros, que observaram uma proporção de $62,3 \%$ de animais reagentes. Da mesma forma, os estudos realizados por Langoni et al. (2000) em bovinos provenientes de diferentes regiões do estado de São Paulo, de Oliveira et al. (2001) e de Aguiar et al (2006), registraram $45,56 \%, 47,63 \%$ e $52,8 \%$ de prevalências, respectivamente. Cabe ressaltar, que neste estudo, as imperfeições dos testes de diagnóstico foram corrigidas quando se calculou a prevalência real de animais $(98,8 \%)$, o que aumentou ainda mais a prevalência.

Na região Centro-Oeste, estudos realizados por Juliano et al. (2000), em bovinos leiteiros na microrregião de Goiânia e por Madruga et al. (1980) em bovinos de corte no estado do Mato Grosso, revelaram prevalência em animais de $81,9 \%$ e $74,5 \%$, respectivamente, semelhantes à obtida neste estudo (Quadro 3), o que sugere que a infecção por Leptospira spp. se mantém prevalente nesta região, tanto na exploração de corte como de leite, há mais de três décadas, sem tendência ao controle.

No presente trabalho, os sorovares Hardjo $(65,6 \%)$ e Wolffi (12,3\%) (Fig.2), embora com prevalências distintas, concordam quanto à predominância com os achados de Favero et al. (2001), em levantamento realizado em 21 estados brasileiros, que encontraram $51,5 \%$ de Hardjo e $24,2 \%$ de Wolffi e de Lilenbaum \& Souza (2003), no Rio de Janeiro, que obtiveram $43,8 \%$ de reações para Hardjo e 24,7\% para Wolffi. Em Minas Gerais, Araújo et al. (2005) encontraram $19,7 \%$ de Hardjo e $13,2 \%$ de Wolffi. No estado da Paraíba (Lage et al. 2007), o sorovar Hardjo foi o mais freqüente com $16,05 \%$ das reações positivas. 
Da mesma forma, os resultados encontrados confirmam achados anteriores em levantamentos realizados por Pellegrin et al. (1992), Pellegrin \& Sereno (1994) e Pellegrin et al. (1999) na região do Pantanal sul-matogrossense, que constataram o sorovar Hardjo como o mais observado, seguido pelo sorovar Wolffi. No estudo de Langoni et al. (2000), em SP, os mesmos sorovares foram encontrados como prevalentes, porém o sorovar Wolffi foi o mais freqüente $(70,59 \%)$ seguido do Hardjo $(67,57 \%)$. Já Homem et al. (2001), em Uruará, encontraram o sorovar Hardjo $(61,2 \%)$ e Bratislava $(9 \%)$ como os mais prevalentes, assim como Oliveira et al. (2001), em Pernambuco, constataram Hardjo em 21,98\% das reações e Bratislava em $15,73 \%$. Na microrregião de Goiânia, GO, Juliano et al. (2000) encontraram o sorovar Wolffi $(36,1 \%)$ como o mais prevalente, seguido dos sorovares Icterohaemorrhagiae $(20,50 \%)$ e Hardjo (5,2\%).

Neste estudo, embora haja predominância dos sorovares Hardjo e Wolffi, também relatada por outros pesquisadores em gado de corte e leite, não deve ser descartada a possibilidade de reação cruzada no sorodiagnóstico, pois ambos os sorovares pertencem ao sorogrupo Sejroe (Costa et al. 1998). Mesmo que tenha sido comprovada por sorodiagnóstico a existência do sorovar Wolffi, a patogenicidade deste não foi comprovada em bovinos, tendo sido experimentalmente verificada em ovinos (Batra et al. 1991).

Dentre os sorovares mais prováveis (dados não mostrados), o Hardjo foi o mais prevalente nos rebanhos de 21 dos 22 municípios amostrados, sendo Douradina, o único a apresentar o sorovar Bratislava como o mais freqüente. Este achado não significa que reações para o sorovar Hardjo não ocorreram, pois em decorrência do critério para seleção dos sorovares mais prováveis (Favero et al. 2001), tais reações foram excluídas. A predominância de reações para o sorovar Hardjo, neste estudo, reforça a teoria de que a espécie bovina é o hospedeiro preferencial para esse sorovar (Ellis 1994, Pellegrin et al. 1999), e que sua disseminação ocorre devido a fatores ambientais ligados ao manejo (Faine 1982).

A identificação do sorovar Hardjo como predominante nos rebanhos indica que estão presentes os mecanismos de transmissão de bovino a bovino. Assim, conforme Lilenbaum (1996), três medidas podem ser praticadas simultaneamente: evitar a introdução de animais no rebanho, salvo quando negativos ao sorodiagnóstico ou previamente tratados com dihidroestretomicina; tratar os animais sororreagentes do rebanho com dihidroestretomicina; fortalecimento da imunidade utilizando uma vacina que contenha as principais variedades presentes na região.

Embora baixa, a prevalência obtida para o sorovar Pomona (Fig.2) deve ser considerada, pois a soropositividade para esse sorovar quando presente pode induzir ao aborto em bovinos. Nessa espécie, título alto é comumente achado no momento do aborto, porque a incidência clínica ocorre durante a fase aguda da infecção. Ao contrário, para o sorovar Hardjo a resposta sorológica no aborto é mais variável, com alguns animais soronegativos e outros mostrando altos títulos (OIE 2006).

O valor preditivo positivo obtido no estrato estudado indica que a probabilidade dos rebanhos diagnosticados como positivos serem falso-positivos é próxima de zero (Quadro 2). Dos rebanhos classificados como negativos, pode-se dizer que estes têm alta probabilidade de serem falso-negativos, como indica o valor preditivo negativo baixo, o que sempre acontece em situações de prevalência muito alta (Noordhuizen et al. 1997).

As análises das variáveis associadas à soropositividade dos rebanhos para pelo menos um sorovar identificaram como fatores de risco o tipo de exploração e a raça. Estas variáveis estão naturalmente associadas, uma vez que a maioria das propriedades de bovinos de corte em sistema extensivo é constituída por animais da raça Nelore. Rebanhos provenientes de exploração do tipo corte (Quadro 4) têm 12,17 vezes mais chances de apresentarem infecção para pelo menos um sorovar, quando comparados com o tipo leite ou com exploração mista (OR=12,73). Vasconcellos et al. (1997) examinando rebanhos leiteiros e de corte, em seis estados brasileiros, observaram uma proporção mais elevada de soropositividade entre os bovinos de corte. Os rebanhos provenientes de animais da raça Zebu (Quadro 5) têm mais chances de apresentarem infecção para pelo menos um sorovar quando comparados aos mestiços $(\mathrm{OR}=11,82)$ e europeu de leite $(\mathrm{OR}=14,11)$. Este achado concorda com o estudo de Prescott et al. (1988) que apontam os rebanhos de exploração do tipo corte como mais suscetíveis em relação aos rebanhos leiteiros e que isto, possivelmente, esteja relacionado com as práticas de manejo, como a monta natural em rebanhos de corte e inseminação artificial em rebanhos de leite.

Contrariamente a este estudo, algumas variáveis relacionadas ao manejo são comumente identificadas como possíveis de aumentar o risco de infecção do rebanho pelo sorovar Hardjo, como: bovinos e ovinos coabitando o mesmo pasto (Lilenbaum \& Souza 2003); acesso de bovinos a fontes de água contaminada, como riachos, rios, alagamentos ou drenagem de água (Bennett 1993, Hunter 2005), além da movimentação de animais (Faine 1982). Tais discrepâncias podem ser explicadas pela alta prevalência obtida neste estudo, onde se observou que a infecção está presente independentemente das variáveis de risco relacionadas com as características de produção e manejo dos animais.

A infecção por Leptospira spp. encontra-se presente em todos os municípios estudados, observando-se alta prevalência tanto em animais como em rebanhos. Apesar de o sorovar Hardjo ser o mais prevalente, não é o único a circular na população bovina da região, devendo ser considerado na adoção de práticas de profilaxia e controle da doença.

\section{REFERÊNCIAS}

Aguiar D.M., Gennari S.M., Cavalcante G.T., Labruna M.B., Vasconcellos S.A., Rodrigues A.A.R., Moraes Z.M. \& Camargo L.M.A. 2006. Sero- 
prevalence of Leptospira spp. in cattle from Monte Negro municipality, western Amazon. Pesq. Vet. Bras. 26(2):102-104.

Araújo V.E.M., Moreira E.C., Navega L.A.B., Silva J.A. \& Contreras R.L. 2005. Freqüência de aglutininas anti-Leptospira interrogans em soros sangüíneos de bovinos, em Minas Gerais, de 1980 a 2002. Arq. Bras. Med. Vet. Zootec. 57(4):430-435.

Batra H., Chandidramani N.K. \& Mandokhot U.V. 1991. Clinical, bacteriological, serological, pathological and metabolic studies of Leptospira interrogans serovar wolffi infection in sheep. Indian J. Anim. Sci. 61(1):6-12.

Bennett R.M. 1993. Decision support models of leptospirosis in dairy herds. Vet. Rec. 132(3):59-61.

Bennett S., Woods T., Liyanage W.M. \& Smith D.L. 1991. A simplified general method for cluster sample surveys of health in developing countries. World Health Statistics Quartely 44(3):98-106.

Blenden D.C. 1976. Aspectos epidemiológicos de la leptospirosis. In: $8^{\text {a }}$ Reunion Interamericana sobre el Controle de la Fiebre Aftosa y otras Zoonosis, Guatemala, 1975. Publicacion Científica 316, Organizacion Panamericana de La Salud, Washington, p.160-168.

Brasil 1995. Manual de Leptospirose. $2^{\underline{a}}$ ed. Fundação Nacional de Saúde, Ministério da Saúde, Brasília. 98p.

Brasil 2002. Resolução no 05 de 10.10.2002. Instituto Brasileiro de Geografia e Estatística (IBGE), Ministério do Planejamento, Orçamento e Gestão, Brasília, DF.

Brasil 2003. Produção da Pecuária Municipal (PPM). Vol.31. Instituto Brasileiro de Geografia e Estatística (IBGE), Ministério do Planejamento, Orçamento e Gestão, Brasília, DF.

Brasil 2004. Produção da Pecuária Municipal (PPM). Vol.32. Instituto Brasileiro de Geografia e Estatística (IBGE), Ministério do Planejamento, Orçamento e Gestão, Brasília, DF.

Cervantes L.P.M., Puebla M.A.C., Rosas D.G., Serranía N.R. \& Barranca J.I.T. 2002. Estudio serológico de leptospirosis bovina en México. Revta Cubana Med. Trop. 54(1):24-27.

Costa M.C.R., Moreira E.C., Leite R.C. \& Martins N.R.S. 1998. Avaliação da imunidade cruzada entre Leptospira hardjo e $L$. wolffi. Arq. Bras. Med. Vet. Zootec. 50(1):11-17.

Cumberland P., Everard C.O.R. \& Levett P.N. 1999. Assessment of the efficacy of an IgM-ELISA and microscopic agglutination test (MAT) in the diagnosis of acute leptospirosis. Am. J. Trop. Med. Hyg. 61(5):731734.

Dohoo I., Martin W. \& Stryhn, H. 2003. Veterinary Epidemiologic Research. AVC Inc., Charlottetown, Prince Edward Island, Canada (www.upei.ca/ver), p.114.

Ellis W.A. 1994. Leptospirosis as a cause of reproductive failure. Vet. Clin. North Am. Food Anim. Pract. 10:463-478.

Faine S. 1982. Guidelines for the Control of Leptospirosis. World Health Organization, Geneva. 171p.

Favero M., Pinheiro S.R., Vasconcellos S.A., Morais Z.M., Ferreira F. \& Ferreira Neto J.S. 2001. Leptospirose bovina: variantes sorológicas predominantes em colheitas efetuadas no período de 1984 a 1997 em rebanhos de 21 estados do Brasil. Arqs Inst. Biológico, São Paulo, 68(2):29-35.

Homem V.S.F., Heinemann M.B., Moraes Z.M., Vasconcellos S.A., Ferreira F. \& Ferreira Neto J.S. 2001. Estudo epidemiológico da leptospirose bovina e humana na Amazônia oriental brasileira. Revta Soc. Bras. Med. Trop. 34(2):173-180.

Hunter P. 2005. Leptospirosis, p.1445-1456. In: Coetzer J.A.W. \& Tustin R.C. (Eds), Infectious Diseases of Livestock. Vol.1. $2^{\text {nd }}$ ed. Oxford University Press, Cape Town.

Juliano R.S., Chaves N.S.T., Santos C.A., Ramos L.S., Santos H.Q., Meireles L.R., Gottschalk S. \& Corrêa Filho R.A.C. 2000. Prevalência e aspectos epidemiológicos da leptospirose bovina em rebanho leiteiro na microrregião de Goiânia, GO. Ciência Rural 30(5):857-862.
Lage A.P., Leite R.M.H., Thompson J.A., Bandeira D.A., Herrmann G.P., Moreira E.C. \& Gonçalves V.S.P. 2007. Serology for Leptospira sp. in cattle of the State of Paraíba, Brazil. Arqs Inst. Biológico, São Paulo, 74(3):185-190.

Langoni H., Meireles L.R., Gottschalk S., Cabral K.G. \& Silva A.V. 2000. Perfil sorológico da leptospirose bovina em regiões do Estado de São Paulo. Arqs Inst. Biológico, São Paulo, 67(1):37-41.

Lilenbaum W. \& Santos M.R.C. 1995. Leptospirosis in animal reproduction: III. Role of the hardjo serovar in bovine leptospirosis in Rio de Janeiro, Brazil. Revta Latinoam. Microbiol. 37(2):87-92.

Lilenbaum W. \& Souza G.N. 2003. Factors associated with bovine leptospirosis in Rio de Janeiro, Brazil. Res. Vet. Sci. 75:249-251.

Lilenbaum W. 1996. Atualização em leptospiroses bovinas. Revta Bras. Med. Vet. 18(1):9-13

Madruga C.R., Aycardi E. \& Putt N. 1980. Freqüência de aglutininas anti-leptospira em bovinos de corte na região Sul de Cerrado do estado de Mato Grosso. Arq. Esc. Vet. UFMG 32(2):245-249.

Martin S.W., Meek A.H. \& Willeberg P. 1987. Veterinary Epidemiology: Principles and methods. lowa State University Press, Ames. 343p.

Martin S.W., Shoukri M. \& Thorburn M.A. 1992. Evaluating the health status of herds based on tests applied to individuals. Prev. Vet. Med. 14:33-43.

Noordhuizen J.P.T.M., Frankena K., Van Der Hoofd C.M., Graaf E.A.M. 1997. Application of Quantitative Methods in Veterinary Epidemiology. Wageningen Pers, Wageningen. 445p.

OIE 2006. Leptospirosis, Chapter 2.2.4. World Organisation for Animal Health. Disponível em: http://www.oie.int/eng/normes/mmanual/ A_00043.htm. Acesso em 6.1.2006.

Oliveira A.A.F., Mota R.A., Pereira G.C., Langoni H., Souza M.I., Navegantes W.A. \& Sa M.E.R. 2001. Seroprevalence of bovine leptospirosis in Garanhuns municipal district, Pernambuco State, Brazil. Onderstepoort J. Vet. Res. 68:275-279.

Pellegrin A.O. \& Sereno J.R.B. 1994. Leptospirose e sua relação com fertilidade em um grupo de matrizes neloradas no Pantanal, sub-região da Nhecolândia. In: Resumos 23ํㅡㄹ. Congr. Bras. Med. Veterinária, Olinda, p.189.

Pellegrin A.O., Guimarães P.H.S., Sereno J.R.B., Figueiredo J.P., Lage A.P., Moreira E.C. \& Leite R.C. 1999. Prevalência da leptospirose em bovinos do Pantanal Mato-Grossense. Comunicado Técnico 22, Embrapa Pantanal, Corumbá, p.1-9.

Pellegrin A.O., Sereno J.R.B. \& Figueiredo J.O. 1992. Levantamento sorológico de aglutininas anti-leptospira em bovinos da sub-região da Nhecolândia, Pantanal Sul-Matogrossense. 22ํㅡ. Congr. Bras. Med. Veterinaria, Curitiba, PR. Socuvet, p.145

Prescott J.F., Miller R.B., Nicholson V.M., Martin S.W. \& Lesnick T. 1988. Seroprevalence and association with abortion of leptospirosis in catle in Ontario. Can. J. Vet. Res. 52:210-215.

Sampaio I.B.M. 2007. Estatística Aplicada à Experimentação Animal. Vol.1. 3를 ed. FEP-MVZ, Belo Horizonte. 264 p.

Thompson J.A., Leite R.M.H., Gonçalves V.S.P., Leite R.C., Bandeira D.A., Herrmann G.P., Moreira E.C., Prado P.E.F., Lobato Z.I.P., Brito C.P.T. \& Lage A.P. 2006. Spatial hierarchical variances and age covariances for seroprevalence to Leptospira interrogans serovar hardjo, BoHV-1 and BVDV for cattle in the State of Paraíba, Brazil. Prev. Vet. Med. 76:290-301

Thrusfield M. 1995. Veterinary Epidemiology. 2nd ed. Blackwell Science, Cambridge. 479 p.

Vasconcellos S.A., Barbarini Júnior O., Umehara O., Morais Z.M., Cortez A., Pinheiro S.R., Ferreira F., Fávero A.C.M. \& Ferreira Neto J.S. 1997. Leptospirose bovina: níveis de ocorrência e sorotipo predominantes em rebanhos dos estados de Minas Gerais, São Paulo, Rio de Janeiro, Paraná, Rio Grande do Sul e Mato Grosso do Sul, no período de janeiro a abril de 1996. Arqs Inst. Biológico, São Paulo, 64:715. 